\title{
Correction to: Continuous translation invariant valuations on convex bodies
}

\author{
by U. BetKe and P. R. GODEY
}

The purpose of this note is to point out an error in our earlier paper [1]. At present it is not clear to us whether the main result, Theorem 1 , of that paper is correct. The error lies in our assumption that the mapping $i$ defined in (14) of [1] is an injection. This assumption is later used in an application of the Hahn-Banach theorem which plays a key role in the proof. At present the best result we can obtain using the original techniques is as follows:

Assume $\varphi$ is a continuous translation invariant valuation on $P^{d}$ which is homogeneous of degree $r$ for some $r \in\{1, \ldots, d-1\}$. Let $\left(P_{i}\right)_{i-1}^{\infty}$ be a sequence of polytopes which is dense in $P^{d}$. There are sequences $\left(L_{n}\right)_{n=1}^{\infty}$ and $\left(M_{n}\right)_{n=1}^{\infty}$ in $K^{d}$ such that

$$
\varphi\left(P_{i}\right)=\lim _{n \rightarrow \infty}\left\{V\left(L_{n} ; P_{i}, r ; B, d-1-r\right)-V\left(M_{n} ; P_{i}, r ; B, d-1-r\right)\right\}
$$

for all $i$.

[1] U. Betke, P. R. Goodey, Continuous translation invariant valutations on convex bodies. Abh. Math. Sem. Hamburg 54 (1984), 95-105.

Ansohrift der Autoren: U. Betke, Mathematisches Institut, Universität Siegen, Hölderlinstr. 3, D-5900 Siegen; P. R. Godey, Department of Mathematics, Oklahoma University, Norman, Oklahoma 73019, USA. 